\title{
Capacity of Deterministic Z-Chain Relay-Interference Network
}

\author{
Soheil Mohajer*, Suhas N. Diggavi*, Christina Fragouli* and David N. C. Tse ${ }^{\dagger}$ \\ * École Polytechnique Fédéral de Lausanne, Lausanne, Switzerland. \\ Email: \{soheil.mohajer, suhas.diggavi, christina.fragouli\}@epfl.ch \\ $\dagger$ Wireless Foundations, UC Berkeley, Berkeley, California, USA. \\ Email: dtse@eecs.berkeley.edu
}

\begin{abstract}
The wireless multiple-unicast problem is considered over a layered network, where the rates of transmission are limited by the relaying and interference effect. The deterministic model introduced in [3] is used to capture the broadcasting and multiple access effects. The capacity region of the $\mathrm{Z}$-chain relayinterference network is fully characterized. In order to solve the problem, we introduce a new achievability scheme based on "interference neutralization" and a new analysis technique to bound the number of non-interfering (pure) signals.
\end{abstract}

\section{INTRODUCTION}

Unlike a wired network, a transmitted signal is broadcast in a wireless system, and hence causes interference between simultaneously transmitted signals. The interference can be used for cooperation, but also causes competition between distinct information flows. Hence, a fundamental question is how to manage interference in a wireless network.

In a general multiple unicast setup, many sources transmit messages, and each is of interest for one of the receivers. The interference channel problem [1] is the very basic example of such situation. The best known achievable region for this problem is due to Han and Kobayashi [1]. Over the past few decades, several techniques have been devised for transmission on the interference channels; among them, superposition of information, power allocation, and interference suppression are the most well-known ones. Recently, the capacity region of the interference channel has been characterized for some regimes by building on an approximate characterization (within 1 bit) given for the whole regime in [2].

The deterministic approach, studied by Avestimehr, Diggavi, and Tse [3], simplifies the wireless network interaction model by eliminating the noise. This approach was successfully applied to the relay network in [3], and resulted in insight in terms of transmission techniques. These insights also led to an approximate characterization of the noisy wireless relay network problem [4].

This model was also applied to a two-layers two-unicast system, called ZZ network in [5] and the capacity region was fully characterized under the deterministic model ${ }^{1}$. Moreover, a new transmission technique called interference neutralization was introduced to deal with the interference. In this technique, the interference caused by the two layers of the network are

\footnotetext{
${ }^{1}$ This idea also gives us insight to obtain an approximate (within constant number of bits) characterization for the Gaussian ZZ network.
}

used to (partially) neutralize each other. It is shown that interference neutralization is crucial to achieve the performance of the network for some regime of parameters.

In this paper, we generalize the above mentioned network, and consider a chain with arbitrary number of $Z$ channels, and characterize the region of the admissible rates under the deterministic model. We first develop a genie-aided outer bound for the rate-region.

We then show that this rate region is achievable using linear operations, where decoding a message of rate $r$ is possible if and only if $r$ non-interfered linearly independent equations describing the message are available at the receiver. In our achievability proof, we use a new technique, called analysis of pure equations, where we keep track of the number of the equations involving bits of each of the interfering messages at the relay nodes in the different layers of the network. We show that among all possible encoding schemes at the layers, there exists at least one which guarantee to provide the desired number of proper equations.

The rest of this paper is organized as follows. We describe the transmission model and the problem in Section II. The outer bound and the achievability analysis are given in Sections III and IV, respectively. Finally we conclude in Section V.

\section{The Problem Statement}

Consider the network shown in Fig. 1, in which the transmitters $S_{1}$ and $S_{2}$ wish to communicate at rates $r_{1}$ and $r_{2}$ to the destination nodes $D_{1}$ and $D_{2}$, respectively. The network is formed by $N$ layers of $\mathrm{Z}$ channels, where the $k$-th one connects the relay nodes $A_{k-1}$ and $B_{k-1}$ to $A_{k}$ and $B_{k}$, for $k=0,2, \ldots, N$, and the relays in layers zero and $N$ are the transmitters and receivers, respectively. We denote the parameters of the network by a family of triples, $\left\{\left(\alpha_{k}, \beta_{k}, \gamma_{k}\right)\right\}_{k=1}^{N}$, where $\alpha_{k}$ and $\beta_{k}$ are the gains of the first and second direct links of the $k$-th layer of the network and $\gamma_{k}$ is the gain of the cross link of the same layer.

We denote the inputs of the layer $k$ by $X_{1}^{(k)}$ and $X_{2}^{(k)}$ and received vectors of this layer by $Y_{1}^{(k)}$ and $Y_{2}^{(k)}$. Transmission model in the $k$-th layer of the Z-chain network can be written 


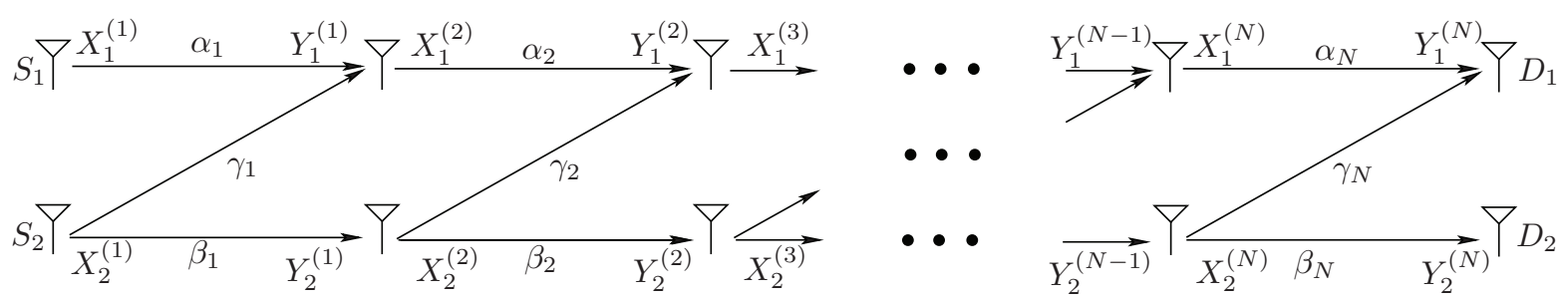

Fig. 1: Transmission model: the Z-chain network.

as

$$
\begin{aligned}
& Y_{1}^{(k)}=N_{11}^{(k)} X_{1}^{(k)}+N_{12}^{(k)} X_{2}^{(k)}, \\
& Y_{2}^{(k)}=N_{22}^{(k)} X_{2}^{(k)},
\end{aligned}
$$

where $\alpha_{k}=\operatorname{rank} N_{11}^{(k)}, \beta_{k}=\operatorname{rank} N_{22}^{(k)}$, and $\gamma_{k}=\operatorname{rank} N_{12}^{(k)}$.

The relay node $A_{k}$ forms its encoded message for the next layer, $X_{1}^{(k+1)}$ as a function of its received signal $Y_{1}^{(k)}$, and similarly for $B_{k}$.

The rate pair $\left(r_{1}, r_{2}\right)$ is called admissible if and only if, for some large enough $n$, there exist codes of length $n$ to be used at the relays such that $W_{1} \in\left\{1, \ldots, 2^{n r_{1}}\right\}$ and $W_{2} \in\left\{1, \ldots, 2^{n r_{2}}\right\}$ can be transmitted to the destination nodes, respectively, with vanishing error probability. Characterization of $\mathcal{R}$, the set of all such rate pairs, is the main question solved in this paper.

\section{The OUter Bound}

The following theorem provides an outer bound for the capacity region by upper bounding the individual rates as well as the sum-rate. The main idea here is to give the relay at the $k$-th layer the interfering signal from all the other layers. This genie-aided side-information is shown to allow the relay node $A_{k}$ to decode message $W_{1}$ if $D_{1}$ is able to do so.

Theorem 1: Let

$$
\begin{aligned}
\mathcal{R}^{U} \triangleq\left\{\left(r_{1}, r_{2}\right):\right. & r_{1} \leq \alpha_{k} \quad \forall k, \\
& r_{2} \leq \beta_{k} \quad \forall k, \\
& \left.r_{1}+r_{2} \leq \Psi_{k}+\Gamma_{N}-2 \gamma_{k} \quad \forall k,\right\}
\end{aligned}
$$

where $\Psi_{k} \triangleq \max \left(\alpha_{k}, \gamma_{k}\right)+\max \left(\beta_{k}, \gamma_{k}\right)$ and $\Gamma_{k} \triangleq \sum_{i=1}^{k} \gamma_{i}$ for $k=1,2, \ldots, N$. Then $\mathcal{R} \subseteq \mathcal{R}^{U}$.

Proof: Let the rate pair $\left(r_{1}, r_{2}\right)$ be achievable using a code of block length $n$. Hence the transmitters encode the messages $W_{1}$ and $W_{2}$ into sequences $X_{1}^{(1)}[1, \ldots, n]$ and $X_{2}^{(1)}[1, \ldots, n]$. The relays in the first layer receive sequences $Y_{1}^{(1)}[1, \ldots, n]$ and $Y_{2}^{(1)}[1, \ldots, n]$, and perform their encoding functions to obtain their transmitting sequences $X_{1}^{(2)}[2, \ldots, n+1]$ and $X_{2}^{(2)}[2, \ldots, n+1]$. Note that the system is casual and $X_{i}^{(2)}[j]$ is only a function of $Y_{i}^{(1)}[1, \ldots, j-1]$. Similarly, the transmitted sequences in the $k$-th layer of the network are $X_{1}^{(k)}[k, \ldots, n+$ $k-1]$ and $X_{2}^{(k)}[k, \ldots, n+k-1]$. We may drop the time indices, and use bold face notation $\mathbf{X}_{i}^{(k)}$ and $\mathbf{Y}_{i}^{(k)}$ to denote the $n$-tuples for brevity.

We will also denote the interference observed by the relay node $A_{k}$ by $t^{(k)}=N_{12}^{(k)} X_{2}^{(k)}$ for $k=1,2, \ldots, N$, and use bold-face symbol $\mathbf{t}^{(k)}$ to denote a block of length $n$.
The proof of the individual rate inequalities goes through the cut-set bounds. More precisely, (1) is just the evaluation of the amount of information can be transmitted through the cut formed by the links $\left\{\gamma_{1}, \ldots, \gamma_{k-1}, \alpha_{k}\right\}$ (the dashed-line cut in Fig. 2). Similarly, the bound in (2) can be obtained by bounding the maximum information can be passed through the cut specified by the links of gains $\left\{\beta_{k}, \gamma_{k+1}, \ldots, \gamma_{N}\right\}$ (the dotted-line cut in Fig. 2). The proofs of these inequalities are straight forward and omitted for brevity. We refer the reader to [6] for more details.

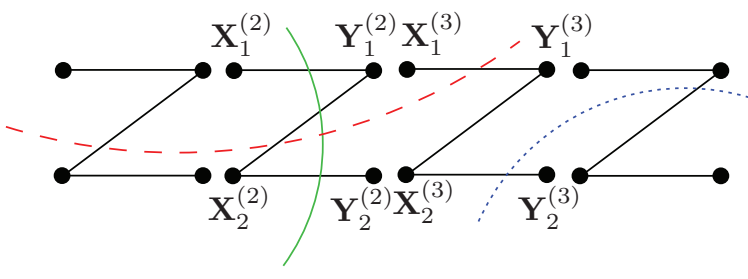

Fig. 2: Three different kinds of cuts

The sum-rate bound is obtained by bounding the amount of information can be passed through the $k$-th layer of the network, namely, the edges $\left\{\alpha_{k}, \beta_{k}, \gamma_{k}\right\}$ (the solid-line cut in Fig.2).

This bound is essentially a genie-aided bound, where a genie provides the output of all the cross links except the $k$-th one $\left(\mathbf{t}^{(1)}, \ldots, \mathbf{t}^{(k-1)}, \mathbf{t}^{(k+1)}, \ldots, \mathbf{t}^{(N)}\right)$ at $A_{k}$. Intuitively, we capture the maximum possible interference neutralization, and argue that having such aid from the genie, $A_{k}$ can decode the message $W_{1}$ if the destination node $D_{1}$ can do.

$$
\begin{aligned}
n\left(r_{1}+\right. & \left.r_{2}\right) \leq I\left(\mathbf{Y}_{1}^{(k)}, \mathbf{Y}_{2}^{(k)} ; \mathbf{X}_{1}^{(k)}, \mathbf{X}_{2}^{(k)}\right) \\
= & H\left(\mathbf{Y}_{1}^{(k)}, \mathbf{Y}_{2}^{(k)}\right) \\
\leq & H\left(\mathbf{Y}_{1}^{(k)}, \mathbf{Y}_{2}^{(k)}, \mathbf{t}^{(1)}, \ldots, \mathbf{t}^{(k-1)}, \mathbf{t}^{(k+1)}, \ldots, \mathbf{t}^{(N)}\right) \\
\leq & H\left(\mathbf{Y}_{1}^{(k)}\right)+H\left(\mathbf{t}^{(1)}\right)+\cdots+H\left(\mathbf{t}^{(k-1)}\right) \\
& +H\left(\mathbf{t}^{(k+1)}\right)+\cdots+H\left(\mathbf{t}^{(N)}\right) \\
& +H\left(\mathbf{Y}_{2}^{(k)} \mid \mathbf{Y}_{1}^{(k)}, \mathcal{T}_{<k}, \mathcal{T}_{>k}\right),
\end{aligned}
$$

where we have used $\mathcal{T}_{<k}=\left(\mathbf{t}^{(1)}, \ldots, \mathbf{t}^{(k-1)}\right)$ and $\mathcal{T}_{>k}=$ $\left(\mathbf{t}^{(k+1)}, \ldots, \mathbf{t}^{(N)}\right)$ for shortening the notations. Note that

$$
\begin{aligned}
H\left(\mathbf{Y}_{1}^{(N)} \mid \mathbf{Y}_{1}^{(k)}, \mathcal{T}_{<k}, \mathcal{T}_{>k}\right) & =H\left(\mathbf{Y}_{1}^{(N)}-\mathbf{t}^{(N)} \mid \mathbf{Y}_{1}^{(k)}, \mathcal{T}_{<k}, \mathcal{T}_{>k}\right) \\
& =H\left(\mathbf{X}_{1}^{(N-1)} \mid \mathbf{Y}_{1}^{(k)}, \mathcal{T}_{<k}, \mathcal{T}_{>k}\right) \\
& \leq H\left(\mathbf{Y}_{1}^{(N-1)} \mid \mathbf{Y}_{1}^{(k)}, \mathcal{T}_{<k}, \mathcal{T}_{>k}\right)
\end{aligned}
$$


Similarly, it can be shown that

$$
H\left(\mathbf{Y}_{1}^{(N)} \mid \mathbf{Y}_{1}^{(k)}, \mathcal{T}_{<k}, \mathcal{T}_{>k}\right) \leq H\left(\mathbf{Y}_{1}^{(k)} \mid \mathbf{Y}_{1}^{(k)}, \mathcal{T}_{>k}\right)=0 .
$$

Combining (5) with Fano's inequality, $H\left(W_{1} \mid \mathbf{Y}_{1}^{(N)}\right) \leq n \varepsilon$, we have

$$
\begin{gathered}
H\left(W_{1} \mid \mathbf{Y}_{1}^{(k)}, \mathcal{T}_{<k}, \mathcal{T}_{>k}\right) \leq H\left(W_{1}, \mathbf{Y}_{1}^{(N)} \mid \mathbf{Y}_{1}^{(k)}, \mathcal{T}_{<k}, \mathcal{T}_{>k}\right) \\
=H\left(\mathbf{Y}_{1}^{(N)} \mid \mathbf{Y}_{1}^{(k)}, \mathcal{T}_{<k}, \mathcal{T}_{>k}\right) \\
\quad+H\left(W_{1} \mid \mathbf{Y}_{1}^{(N)}, \mathbf{Y}_{1}^{(k)}, \mathcal{T}_{<k}, \mathcal{T}_{>k}\right) \leq n \varepsilon
\end{gathered}
$$

We also have

$$
\begin{aligned}
H\left(\mathbf{X}_{1}^{(k)} \mid \mathbf{Y}_{1}^{(k)}, \mathcal{T}_{<k},\right. & \left.\mathcal{T}_{>k}\right) \leq H\left(\mathbf{Y}_{1}^{(k-1)} \mid \mathbf{Y}_{1}^{(k)}, \mathcal{T}_{<k}, \mathcal{T}_{>k}\right) \\
& =H\left(\mathbf{Y}_{1}^{(k-1)}-\mathbf{t}^{(k-1)} \mid \mathbf{Y}_{1}^{(k)}, \mathcal{T}_{<k}, \mathcal{T}_{>k}\right) \\
& =H\left(\mathbf{X}_{1}^{(k-1)} \mid \mathbf{Y}_{1}^{(k)}, \mathcal{T}_{<k}, \mathcal{T}_{>k}\right) .
\end{aligned}
$$

Similarly,

$$
\begin{aligned}
H\left(\mathbf{X}_{1}^{(k)} \mid \mathbf{Y}_{1}^{(k)}, \mathcal{T}_{<k}, \mathcal{T}_{>k}\right) & \leq H\left(\mathbf{X}_{1}^{(1)} \mid \mathbf{Y}_{1}^{(k)}, \mathcal{T}_{<k}, \mathcal{T}_{>k}\right) \\
& \leq H\left(W_{1} \mid \mathbf{Y}_{1}^{(k)}, \mathcal{T}_{<k}, \mathcal{T}_{>k}\right) \leq n \varepsilon
\end{aligned}
$$

where the last inequality is due to (6). Hence,

$$
\begin{aligned}
H\left(\mathbf{t}^{(k)} \mid \mathbf{Y}_{1}^{(k)}, \mathcal{T}_{<k}, \mathcal{T}_{>k}\right) \leq H\left(\mathbf{t}^{(k)}, \mathbf{X}_{1}^{(k)} \mid \mathbf{Y}_{1}^{(k)}, \mathcal{T}_{<k}, \mathcal{T}_{>k}\right) \\
=H\left(\mathbf{X}_{1}^{(k)} \mid \mathbf{Y}_{1}^{(k)}, \mathcal{T}_{<k}, \mathcal{T}_{>k}\right) \\
\quad+H\left(\mathbf{t}^{(k)} \mid \mathbf{Y}_{1}^{(k)}, \mathbf{X}_{1}^{(k)}, \mathcal{T}_{<k}, \mathcal{T}_{>k}\right) \\
\leq n \varepsilon .
\end{aligned}
$$

where the last is zero since $\mathbf{t}^{(k)}=\mathbf{Y}_{1}^{(k)}-N_{11}^{(k)} \mathbf{X}_{1}^{(k)}$. Finally,

$$
\begin{aligned}
H\left(\mathbf{Y}_{2}^{(k)} \mid \mathbf{Y}_{1}^{(k)}, \mathcal{T}_{<k}, \mathcal{T}_{>k}\right) \leq & H\left(\mathbf{Y}_{2}^{(k)}, \mathbf{t}^{(k)} \mid \mathbf{Y}_{1}^{(k)}, \mathcal{T}_{<k}, \mathcal{T}_{>k}\right) \\
= & H\left(\mathbf{t}^{(k)} \mid \mathbf{Y}_{1}^{(k)}, \mathcal{T}_{<k}, \mathcal{T}_{>k}\right) \\
& +H\left(\mathbf{Y}_{2}^{(k)} \mid \mathbf{Y}_{1}^{(k)}, \mathcal{T}_{<k}, \mathbf{t}^{(k)}, \mathcal{T}_{>k}\right) \\
\leq & n \varepsilon+H\left(\mathbf{Y}_{2}^{(k)} \mid \mathbf{t}^{(k)}\right) \\
\leq & n\left(\beta_{k}-\gamma_{k}\right)^{+}+n \varepsilon
\end{aligned}
$$

Replacing (9) in (4) we get

$n\left(r_{1}+r_{2}\right) \leq n \max \left(\alpha_{k}, \gamma_{k}\right)+\sum_{i \neq k} n \gamma_{i}+n\left(\beta_{k}-\gamma_{k}\right)^{+}+n \varepsilon^{\prime}$

which yields in (3) after some simplifications.

\section{ACHiEVABILITy}

The goal of this section is to show that any rate pair $\left(r_{1}, r_{2}\right) \in \mathcal{R}^{U}$ is achievable. We will show that such rate pair is achievable using only linear operations, hence, the signal at any relay or destination node would be a linear combination of the input bits of $W_{1}=\left[b_{1}(1), b_{1}(2), \ldots, b_{1}\left(r_{1}\right)\right]^{T}$ and $W_{2}=\left[b_{2}(1), b_{2}(2), \ldots, b_{2}\left(r_{2}\right)\right]^{T}$, the binary representations of the input messages. It is clear that destinations can decode if and only if the nodes $D_{1}\left(D_{2}\right)$ can obtain exactly $r_{1}$ $\left(r_{2}\right)$ linearly independent equations which only involve the unknown bits of $W_{1}\left(W_{2}\right)$ from the set of received equations. In order to show achievability, we introduce a new interference management scheme we term interference neutralization. Here the interfering signal is eliminated when mixed over the air, without necessarily decoding it. This was also used in the ZZ network in [5], and is crucial in this work as well.

We focus on a special class of encoding schemes, where the relay nodes $B_{k}$ 's, first decode the corresponding message $W_{2}$, and then encode it again and send exactly $r_{2}$ linearly independent equations describing $W_{2}$. The encoding scheme at $B_{k}$ can be chosen such that the message received at $A_{k+1}$ gets more interference, or (a part of) its interference get neutralized. We choose $r_{2}$ nodes among the top $\beta_{k}$ available nodes for transmission, opportunistically, such that the message can be decoded at $B_{k+1}$ and the desired interfering situation happens at $A_{k+1}$.

Also the relay nodes $A_{k}$ transmit exactly $r_{1}$ equations, where some of them may only involve bits from $W_{1}$ and the others involve bits of both $W_{1}$ and $W_{2}$. However, the equations are chosen such that the induced equations on each of $W_{1}$ and $W_{2}$ are linearly independent, i.e., it transmits a vector

$$
X_{1}^{(k)}=\left[\begin{array}{c|c}
U & Q \\
\hline V & 0
\end{array}\right]\left[\begin{array}{l}
W_{1} \\
\hline W_{2}
\end{array}\right]
$$

where the matrices $Q$ and $\left[\begin{array}{cc}U^{T} & V^{T}\end{array}\right]^{T}$ are full-rank.

Transmission of $W_{2}$ from $S_{2}$ to $D_{2}$ through $B_{k}$ needs to only send linearly $r_{2}$ independent equations since no interference can affect the message. We call a linear equation pure if it only involves the bits of $W_{1}$ as the unknown variables. Let $p_{k}$ denote the number of linearly independent pure equations received at the relay node $A_{k}$ for $k=1, \ldots, N-1$. Similarly $p_{0}=r_{1}$ and $p_{N}$ denote the number of pure equations at $S_{1}$ and $D_{1}$, respectively. In fact $A_{k}$ has $p_{k}$ pure equations and $r_{1}-p_{k}$ mixed equations involving the unknown bits of both $W_{1}$ and $W_{2}$. It may also have some equations which only involve unknown bits of $W_{2}$. Such equations can be used for interference suppression as well as interference neutralization.

The value of $p_{k}$ depends on both the number of pure equation in the previous layer, $p_{k-1}$, as well the encoding strategy used at the relay nodes $A_{k-1}$ and $B_{k-1}$. Therefore, even for a fixed $p_{k-1}$, different values for $p_{k}$ can be obtained using different coding strategies. In the following we will study the evolution of the number of pure equations and show that if $\left(r_{1}, r_{2}\right) \in \mathcal{R}^{U}$ then there exist coding strategies used at the relays such that one can obtain $p_{N}=r_{1}$ pure equations at $D_{1}$ . It is clear that having $r_{1}$ linearly independent equations, $D_{1}$ can reconstruct the bits of $W_{1}$.

Define $\mathcal{P}_{k}$ as the set of all possible number of pure equations at the $k$-th layer of the network. We define $M_{k}=\max \mathcal{P}_{k}$ and $m_{k}=\min \mathcal{P}_{k}$ as the largest and smallest elements of $\mathcal{P}_{k}$, respectively. It is clear that $M_{k} \leq r_{1}$ since the number of linearly independent equations cannot exceed the number of variables. Also note that we never send more than $r_{2}$ equations from $B_{k-1}$, and therefore the number of mixed equations cannot exceed $r_{2}$. Therefore, we have $r_{1}-p_{k} \geq r_{2}$ and therefore $m_{k} \geq\left(r_{1}-r_{2}\right)^{+}$.

In the following subsection we will investigate how the $p_{k}$ changes from one layer of the network to the next one. 


\section{A. Evolution of the number of pure equations}

Assume we are at the encoding part of the $k$-th layer of the network with parameters $\left(\alpha_{k}, \beta_{k}, \gamma_{k}\right)$, and have $p_{k-1}$ pure and $r_{1}-p_{k-1}$ mixed equations. We need to find $m_{k}$ and $M_{k}$ that can be achieved for the next layer.

Lemma 1: Given $p_{k-1}$ linearly independent equations at the relay node $A_{k-1}$, the minimum number of pure equations achievable at $A_{k}$ is

$$
\min p_{k \mid p_{k-1}}=\max \left\{0, r_{1}-r_{2}, p_{k-1}-\gamma_{k}\right\} \text {. }
$$

Proof: Minimizing the number of pure equations is equivalent to maximizing the number of mixed equations. A mixed equations at $A_{k}$ can be obtained by either receiving a mixed equations from $A_{k-1}$ whose interference is not neutralized by the new interference, or combination of a pure equation from $A_{k-1}$ and an interference from $B_{k-1}$. Note that we have $r_{1}-p_{k-1}$ mixed equations, and among the $p_{k-1}$ pure equations at most $\gamma_{k}$ of them can become mixed in the next layer. However, since $W_{2}$ has only $r_{2}$ bits, at most $r_{2}$ equations can be affected by the interference. Therefore, the maximum number of mixed equations would be

$$
\begin{aligned}
\max \left(r_{1}-p_{k}\right) & =\min \left\{r_{1}-p_{k-1}+\min \left\{p_{k-1}, \gamma_{k}\right\}, r_{2}\right\} \\
& =\min \left\{r_{1}, r_{1}-p_{k-1}+\gamma_{k} \cdot r_{2}\right\} .
\end{aligned}
$$

Hence $\min p_{k \mid p_{k-1}}=\max \left\{0, r_{1}-r_{2}, p_{k-1}-\gamma_{k}\right\}$.

Lemma 2: If the relay node $A_{k-1}$ sends $p_{k-1}$ pure equations, then maximum achievable number of pure equations in the next layer's relay, $A_{k}$ is

$\max p_{k \mid p_{k-1}}=\min \left\{r_{1}, \Psi_{k}-r_{2}+r_{1}-\gamma_{k}-p_{k-1}, p_{k-1}+\gamma_{k}\right\}$.

Proof: A pure equation at the next layer $A_{k}$ can be obtained by either receiving a pure equation from $A_{k-1}$ at a sub-node which is not affected by the message from $B_{k-1}$, or a mixed message from $A_{k-1}$ whose interference is neutralized ${ }^{2}$ by another equation received from $B_{k-1}$. We denote the number of these two sets of equations by $p_{k}(\mathrm{P})$ and $p_{k}(\mathrm{~N})$, respectively.

We first enumerate the first kind of such messages. The maximum number of sub-nodes in $A_{k}$ which can receive message from $A_{k-1}$ and have not occupied by the signal received from $B_{k-1}$ can be found as illustrated in Fig. 3. The relay node $B_{k-1}$ chooses $r_{2}$ sub-nodes among its top $\beta_{k}$ sub-nodes to transmit its message to $B_{k}$, and at least $r_{2}-\left(\beta_{k}-\gamma_{k}\right)^{+}$of them would be among the top $\gamma_{k}$ subnodes, whose message will be also observed by $A_{k}$. Among them, at most $\left(\gamma_{k}-\alpha_{k}\right)$ are out of the range of $A_{k-1}$, but the remaining will have overlap with the sub-nodes in range of $A_{k-1}$. Therefore, at least $\left[r_{2}-\left(\beta_{k}-\gamma_{k}\right)^{+}-\left(\gamma_{k}-\alpha_{k}\right)^{+}\right]^{+}$ sub-nodes among the $\alpha_{k}$ sub-nodes of $A_{k}$ get interfered.

\footnotetext{
${ }^{2}$ This is done by pre-coding at $B_{k-1}$. Since it can decode the whole vector $\mathbf{X}_{2}$, it can encode it again such that a desired number of mixed equations get neutralized.
}

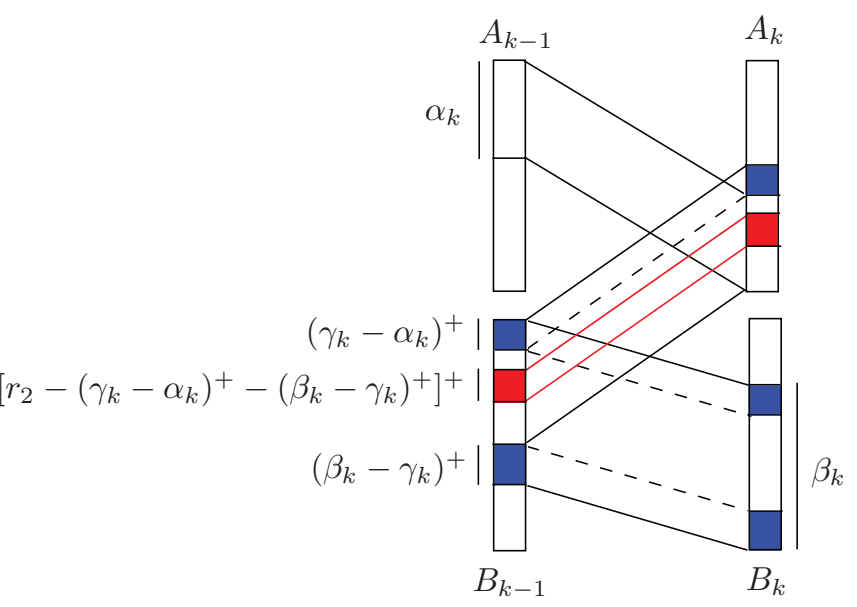

Fig. 3: The maximum number of nodes in $A_{k}$ which are not affected by interference.

Hence, the maximum number of pure equations of the first kind would be

$$
\begin{aligned}
p_{k}(\mathrm{P}) & =\min \left\{p_{k-1}, \alpha_{k}-\left[r_{2}-\left(\beta_{k}-\gamma_{k}\right)^{+}-\left(\gamma_{k}-\alpha_{k}\right)^{+}\right]^{+}\right\} \\
& =\min \left\{p_{k-1}, \alpha_{k}-\left[r_{2}+\alpha_{k}+\gamma_{k}-\Psi_{k}\right]^{+}\right\}
\end{aligned}
$$

On the other hand, we have $r_{1}-p_{k-1}$ mixed equations, where at most $\gamma_{k}$ of them can be neutralized by the message from $B_{k-1}$. Thus, the maximum number of the second class of pure equations at the $k$-th layer would be

$$
p_{k}(\mathrm{~N})=\min \left\{r_{1}-p_{k-1}, \gamma_{k}\right\} \text {. }
$$

By adding up (11) and (12) we have

$$
\begin{aligned}
& \max p_{k \mid p_{k-1}}= \min \left\{p_{k-1}, \alpha_{k}-\left[r_{2}+\alpha_{k}+\gamma_{k}-\Psi_{k}\right]^{+}\right\} \\
&+\min \left\{r_{1}-p_{k-1}, \gamma_{k}\right\} \\
&=\min \left\{r_{1}, \Psi_{k}-r_{2}+r_{1}-\gamma_{k}-p_{k-1}, p_{k-1}+\gamma_{k}\right\}
\end{aligned}
$$

where we have used the fact that $p_{k-1} \leq r_{1} \leq \alpha_{k}$, and $\Psi_{k} \geq$ $\alpha_{k}+\beta_{k} \geq r_{1}+r_{2}$ to simplify the minimization expression.

Note that this value is always achievable by choosing the transmitting nodes of $B_{k-1}$ and the equations they send properly, such that the required number of nodes $A_{k}$ do not affected by interference and a specific number of the rest get neutralized.

The following theorem summarizes the discussion of this subsection.

Theorem 2: The set of all achievable numbers of linearly independent pure equations at the $k$-th layer of the network is

$$
\mathcal{P}_{k}=\left\{p \in \mathbb{Z}^{+}: m_{k} \leq p \leq M_{k}\right\}
$$

where

$$
m_{k}=\max \left\{0, r_{1}-r_{2}, r_{1}-\Gamma_{k}\right\}, \quad k=1,2, \ldots, N,
$$

and $M_{k}$ is obtained using the recurrence relations

$$
M_{k}=\min \left\{r_{1}, \Psi_{k}+\Gamma_{k}-2 \gamma_{k}-r_{2}, \gamma_{k}+M_{k-1}\right\},
$$


for $k=1,2, \ldots, N$ and with the initial condition $M_{0}=r_{1}$.

Proof: The Lemmas 1 and 2 determine the minimum and maximum achievable $p_{k}$ provided that the relay node $A_{k-1}$ sends $p_{k-1}$ pure equations. A similar argument shows that the extreme values in both lemmas are in fact achievable. Not surprisingly, it can shown that if $p_{k}=u_{1}$ and $p_{k}=u_{2}$ are achievable for $u_{1}<u_{2}$, then any integer $u \in\left[u_{1}, u_{2}\right]$ is also achievable.

The minimum and maximum values obtained in Lemmas 1 and 2 depend on $p_{k-1}$. However depending on the required $p_{k}$ at the $k$-layer, one can choose any $p_{k-1} \in \mathcal{P}_{k-1}$ for encoding at $A_{k-1}$. We will prove (15) using induction over $k$. For $k=1$, the claim is just rewriting Lemma 1 since $m_{0}=r_{1}$. Assuming (15) for $k-1$, we have

$$
\begin{aligned}
m_{k} & =\min _{p_{k-1} \in \mathcal{P}_{k-1}} \max \left\{0, r_{1}-r_{2}, p_{k-1}-\gamma_{k}\right\} \\
& =\max \left\{0, r_{1}-r_{2}, m_{k-1}-\gamma_{k}\right\} \\
& =\max \left\{0, r_{1}-r_{2}, r_{1}-\Gamma_{k}\right\} .
\end{aligned}
$$

Similarly, using (13) $M_{k}$ can be obtained as

$$
\begin{aligned}
& \max _{p_{k-1} \in \mathcal{P}_{k-1}} \min \left\{r_{1}, r_{1}+\Psi_{k}-r_{2}-\gamma_{k}-p_{k-1}, \gamma_{k}+p_{k-1}\right\} \\
& =\min \left\{r_{1}, r_{1}+\Psi_{k}-r_{2}-\gamma_{k}-m_{k-1}, \gamma_{k}+M_{k-1}\right\} .
\end{aligned}
$$

Replacing $m_{k}$ from (15), we get

$$
\begin{aligned}
r_{1} & +\Psi_{k}-r_{2}-\gamma_{k}-m_{k-1} \\
& =\min \left\{r_{1}+\Psi_{k}-r_{2}-\gamma_{k}, \Psi_{k}-\gamma_{k}, \Psi_{k}+\Gamma_{k}-2 \gamma_{k}-r_{2}\right\}
\end{aligned}
$$

where the first two terms are not less that $r_{1}$. Therefore

$$
M_{k}=\min \left\{r_{1}, \Psi_{k}+\Gamma_{k}-2 \gamma_{k}-r_{2}, \gamma_{k}+M_{k-1}\right\}
$$

However, solving the last recursive relation and evaluating $M_{k}$ is not easy and we leave it as an optimization expression.

\section{B. An achievable path for $p_{N}=r_{1}$}

Next we show implicitly that for $\left(r_{1}, r_{2}\right)$ satisfying the outer bound, $r_{1} \in \mathcal{P}_{N}$, using the recursive form of the evolution of pure equations obtained in the last subsection. Therefore, this shows that rate $\left(r_{1}, r_{2}\right)$ is indeed achievable.

Lemma 3: Let

$$
p_{k}^{*}=\min \left(r_{1}, \min _{1 \leq \ell \leq k}\left\{\Psi_{\ell}+\Gamma_{k}-2 \gamma_{\ell}-r_{2}\right\}\right) .
$$

Then $p_{k}^{*} \in \mathcal{P}_{k}$ for $k=1,2, \ldots, N$.

Proof: In order to prove the lemma, we need to show $m_{k} \leq p_{k}^{*} \leq M_{k}$. The first inequality is straight forward and shown as follows.

$$
\begin{aligned}
p_{k}^{*}-m_{k}= & \min \left(r_{1}, \min _{1 \leq \ell \leq k}\left\{\Psi_{\ell}+\Gamma_{k}-2 \gamma_{\ell}-r_{2}\right\}\right) \\
& -\max \left(0, r_{1}-r_{2}, r_{1}-\Gamma_{k}\right) \\
= & \min \left\{r_{1}, r_{2}, \Gamma_{k}, \min _{1 \leq \ell \leq k}\left\{\Psi_{\ell}+\Gamma_{k}-2 \gamma_{\ell}-r_{2}\right\},\right. \\
\min _{1 \leq \ell \leq k}\left\{\Psi_{\ell}+\Gamma_{k}-2 \gamma_{\ell}-r_{1}\right\}, & \left.\min _{1 \leq \ell \leq k}\left\{\Psi_{\ell}+2 \Gamma_{k}-2 \gamma_{\ell}-r_{1}-r_{2}\right\}\right\} \geq 0
\end{aligned}
$$

where the last inequality follows from the facts that $r_{1} \leq \alpha_{\ell}$ and $r_{2} \leq \beta_{\ell}$ for $\ell=1, \ldots, N$.

To show the second inequality we use induction over $k$, namely, we show that $p_{k}^{*} \leq M_{k}$ provided that $p_{k-1}^{*} \leq M_{k-1}$. For $k=1$, is claim is trivial by just comparing $p_{1}^{*}$ and $M_{1}$ in (16). Assuming $p_{k-1}^{*} \leq M_{k-1}$ and using (16), we have

$$
\begin{aligned}
& M_{k} \geq \min \left\{r_{1}, \Psi_{k}+\Gamma_{k}-2 \gamma_{k}-r_{2}, \gamma_{k}+p_{k-1}^{*}\right\} \\
&=\min \left\{r_{1}, \Psi_{k}+\Gamma_{k}-2 \gamma_{k}-r_{2},\right. \\
&\left.\min _{1 \leq \ell \leq k-1}\left\{\Psi_{\ell}+\Gamma_{k}-2 \gamma_{\ell}-r_{2}\right\}\right\} \\
&= \min \left\{r_{1}, \min _{1 \leq \ell \leq k}\left\{\Psi_{\ell}+\Gamma_{k}-2 \gamma_{\ell}-r_{2}\right\}\right\} \\
&= p_{k}^{*} .
\end{aligned}
$$

which shows that $p_{k}^{*}$ does not exceed $M_{k}$. Hence $p_{k}^{*} \in \mathcal{P}_{k}$.

Lemma 4:

$$
p_{N}^{*}=r_{1}
$$

Proof: Note that by definition

$$
p_{N}^{*}=\min \left(r_{1}, \min _{1 \leq \ell \leq N}\left\{\Psi_{\ell}-2 \gamma_{\ell}+\Gamma_{N}-r_{2}\right\}\right) \text {. }
$$

In order to prove the lemma one has to show that $\Psi_{\ell}-2 \gamma_{\ell}+$ $\Gamma_{N}-r_{2} \geq r_{1}$ for $1 \leq \ell \leq N$. But such inequality always holds since $\left(r_{1}, r_{2}\right)$ satisfy (3).

Lemmas 3 and 4 together show that $r_{1} \in \mathcal{P}_{N}$, i.e., there exist encoding schemes used at the relays which can provide $r_{1}$ linearly independent pure equations for $A_{N}=D_{1}$. Then it is clear that $D_{1}$ can use such equations to solve for the bits of $W_{1}$.

\section{CONClusion}

We studied the deterministic Z-chain network and fully characterized the capacity region of the network. We believe that the analysis technique of bounding the number of pure signals (equations) would be useful for many other interference network problems.

\section{REFERENCES}

[1] T. S. Han and K. Kobayashi, "A new achievable rate region for the interference channel," IEEE Trans. Inform. Theory, vol. 27, no. 1, pp. 49 60, Jan. 1981.

[2] R. Etkin, D. Tse, and H. Wang, "Gaussian interference channel capacity to within one bit," submitted to the IEEE Trans. Inform. Theory, Feb. 2007.

[3] S. Avestimehr, S. N. Diggavi, and D. N. C. Tse, "A Deterministic approach to wireless relay networks," Proceedings of Allerton Conference on Communication, Control, and Computing, Illinois, September 2007.

[4] S. Avestimehr, S. N. Diggavi, and D. N. C. Tse, "Approximate capacity of Gaussian relay networks," Proceeding of IEEE International Symposium on Information Theory, Toronto, July 2008.

[5] S. Mohajer, S. N. Diggavi, C. Fragouli, and D. N. C. Tse, “ Transmission techniques for relay-interference networks," Proceedings of Allerton Conference on Communication, Control, and Computing, Illinois, September 2008.

[6] S. Mohajer, S. N. Diggavi, C. Fragouli, and D. N. C. Tse, "Capacity of deterministic Z-chain relay-Interference network," EPFL Technical Report, Available at http://infoscience.epfl.ch 